\title{
The Genetics and Learning Disorders of the Syndromes Involving Craniosynostosis
}

\author{
Sarabjot Singh-Makkar ${ }^{1}$, Talwinder Kaur Grewal ${ }^{1}$, Japjeet Kaur ${ }^{1}$, Anjali Sharma ${ }^{1}$, Trissa Paul ${ }^{1}$, Harsimrat Kaur ${ }^{1}$ \\ and Pamela Youssef ${ }^{2}$ \\ ${ }^{1}$ Larkin Health System, South Miami FL, USA \\ ${ }^{2}$ Larkin University, South Miami FL, USA
}

*Corresponding author: Sarabjot Singh Makkar, Larkin Health System, 7031 S.W. 62nd Avenue, South Miami, FL 33143, USA, E-mail: sarabjot.makkar@case.edu

\section{ABSTRACT}

Keywords

Prenatal genetic vehicles that lead to facial and cranial dysmorphias, specifically Craniosynostosis craniosynostosis, are seen in a spectrum of synostotic syndromes that include apert, ADHD crouzon, Kleeblattschadel deformity, saethre-chotzen, muenke, cranio-fronto-nasal syndrome, Robinow-Sorauf syndrome and beare-stevenson-cutis-gyrata syndrome. Specific genes involved in cranio-synostotic syndromes include: TWIST1, EFNB1, GLI2, DMD, YWHAE, IRAK2, FGFR1, FGFR2, FGFR3, CNTNAP2, ADAMTS18, SKI, MECP2, KIFBP, TCF12, H2AL1P, GAGE12D and possibly HDAC9. Regarding protein expression, conserved domains found in rpsblast for craniosynostosis using the NCBI homologene tool show IGc2 (smart00408) immunoglobulin C-2 Type, PKc_like (c109925) Protein Kinases, catalytic domain, Ig (cl11960) Immunoglobulin domain. A discussion of all the syndromes involving craniosynostosis is beyond the scope of this paper. We will discuss the clinical features, genetics, cognitive development and associated psychiatric conditions of the more common syndromes involving craniosynostosis. We theorize that the clinical features and genetics of craniosynostosis involve a spectrum of syndromes on which there is variable severity and involvement of impaired cognition and developmental disorders.

\section{GENETIC LOCI OF CRANIOSYNOSTOSIS SYNDROMES}

Specific genes involved in cranio-synostotic syndromes include: TWIST1, EFNB1, GLI2, DMD, YWHAE, IRAK2, FGFR1, FGFR2, FGFR3, CNTNAP2, ADAMTS18, SKI, MECP2, KIFBP, TCF12, H2AL1P, GAGE12D and possibly HDAC9 [1-6]. TWIST1 is seen in Saethre-Chotzen syndrome. Apert, Beare-Stevenson and Crouzon have FGFR2 mutation; whereas Pfeiffer is complicated by the presence of both FGFR1 and FGFR2 mutations (Figure 1). FGFR3 mutations play a role in Muenke syndrome [1-8]. Regarding protein expression, conserved domains were found in rpsblast for craniosynostosis using the NCBI homologene tool and show IGc2 (smart00408) immunoglobulin C-2 Type, PKc_like(c109925) Protein Kinases, catalytic domain, Ig (cl11960) Immunoglobulin domain [1-3].

These syndromic cranio-synostoses may present with mild early sutural fusion to severe turribrachycephaly (upward extension of the skull deformity) [8,10]. Imaging techniques (3D-CT, MRI) depict several classic features of trigonocephaly (Figure 2). Notably, these include epicanthal folds, the surprised coon sign and a normal cephalic index. The surprised coon sign refers to teardrop-shaped orbits angulated towards the midline of the forehead seen in severe cases [1-8].

A study in Hungary showed that the most frequently involved suture in craniosynostosis is the sagittal suture [9]. A 5-year retrospective study in japan found a 79\% survival rate of 


\section{Review Article}

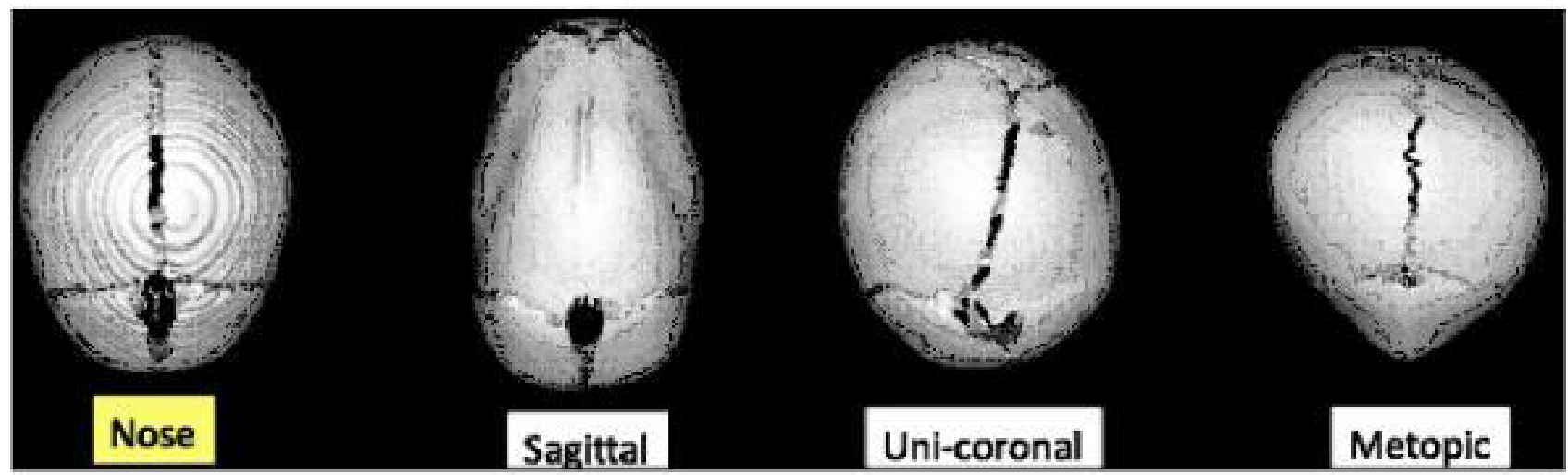

\section{Shapes of unaffected (left), sagittal, uni-coronal and metopic synostosis skulls.}

Figure 1: Schematic of selected craniosynostoses [8].

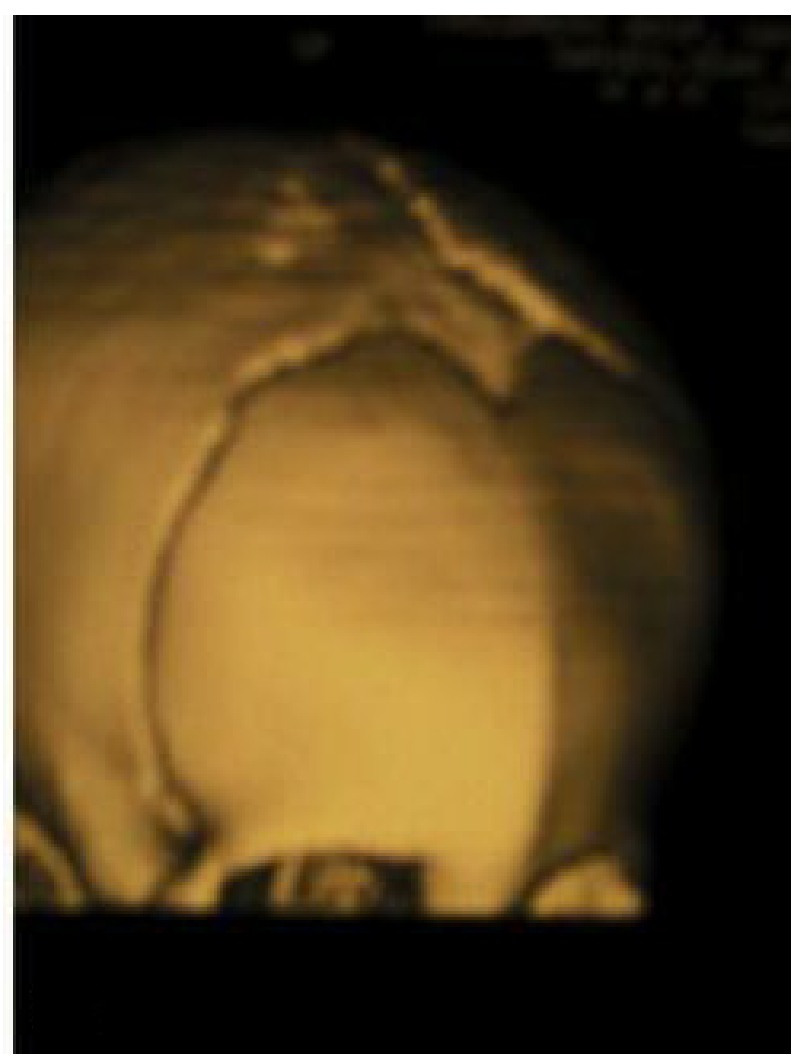

Figure 2: A 3D view depicting trigonocephaly and metopic suture fusion is shown [10].

infants with syndromic craniosynostosis. The ultrasound detection rate was $61 \%$ and the most common syndrome found was Pfeiffer Syndrome [10].

Crouzon syndrome is a milder craniosynostotic syndrome. Crouzon syndrome, also known as craniofacial dysostosis, has an autosomal dominant pattern of inheritance. This syndrome involves missense mutation in the fibroblast growth factor receptor (FGFR $2 \& 3$ ) on chromosome 10q26.13 [11-13]. Downstream signaling effects increase differentiation of osteoblasts $[14,15]$. Crouzon syndrome may be caused by the C342R mutation in FGFR2 according to a study done on Chinese individuals [16]. De novo mutations are the root cause of about $50 \%$ of the cases [17]. Epidemiologically, Crouzon syndrome occurs in 1 in 60,000 newborns. Thus, it is one of the most common craniosynostosis syndromes, a close runner up to Muenke syndrome [18,19].

Crouzon syndrome has complete penetration; however, it has a spectrum of neurological presentations ranging from Kleeblattschadel (cloverleaf skull) with severe intellectual impairment and hydrocephalus to normal development. The expressivity ranges from mild to severe, depending on the phenotype present. Proptosis is a typical finding in this disease. Trigonocephaly and scaphocephaly are not unusual to see; however, brachycephaly is the most likely outcome due to bicoronal fusion of the suture lines in Crouzon syndrome. The most common form of craniosynostosis in Crouzon syndrome is pansynostosis. If there is midline suture synostosis involvement, the cranial fossa volumes may be reduced [20].

Kleeblattschadel deformity is at the critical end of the spectrum, where many suture lines fuse together creating a cloverleaf skull shape. Crouzon syndrome patients could potentially lead life with normal cognitive function, remarkable auditory/ visual performance, etc. if the premature fusion were corrected early [21].

Beare-stevenson-cutis-gyrata syndrome has been identified in 20 people globally. It presents with cutis gyrata and craniosynostosis. Cutis gyrata creates chasms and ripples of 
the scalp's skin that are akin to gyri. These are classically located in the supra-auricular regions and on the palms and soles. Specific cranial features involve a cloverleaf-shaped (also termed as tri-pronged) head, hyper-teloric eyes with proptosis, choanal atresia and micrognathia [8,10,22,23]. Beare-Stevenson syndrome patients have been shown to have mutations in exons 8 and 11 of FGFR2.

Beare-Stevenson syndrome and Crouzon syndrome patients share a mutation: (c.799T $>$ C; p.S267P); however, there is a mutation that is unique to Beare-Stevenson syndrome patients: an in-frame deletion (c.820 824delinsTT; p.V274 Q275delinsL) [24].

Saethre-Chotzen syndrome is seen in 1 in 25,000 people. Sathre-Chotzen syndrome has genetic involvement in both the TWIST1 gene and the EFNB1 gene [25]. Characteristic features in the extremities include webbing between the second and third fingers, and a broad or duplicated hallux. Facial defects include asymmetry and a broad nasal bridge, in addition to a low hairline. The eyes show ptosis and hypertelorism. Microtia can be present, which can result in conductive hearing loss. Conductive hearing loss with both receptive and expressive language delay is common in patients diagnosed with SaethreChotzen. There are two types of Saethre-Chotzen syndrome: deletion vs non-deletional mutation. A genetic deletion of the TWIST gene increases the likelihood of cognitive impairment; whereas, the classic form of Saethre-Chotzen involving a nondeletional mutation does not typically cause developmental delay or intellectual impairment.

Translocations or formation of ring chromosomes can alter or delete the TWIST1 gene in the p arm of chromosome 7, causing Sathre-Chotzen syndrome, which typically causes fusion of the calvaria along the fronto-temporal suture. The TWIST1 protein is activated in part by $\mathrm{PI} 3 \mathrm{~K}$ and functions as a basic helix loop helix (bHLH) structural motif of the transcription factor that controls limb development and bone formation by regulating FGFR2.

The EFNB1 gene is involved in both Saethre-Chotzen syndrome and in cranio-fronto-nasal syndrome (CFNS). The EFNB1 gene is located on chromosome Xq13.1 [26]. CFNS presents with craniosynostosis, anomalies of the fingers, dysplastic nares and a bifid nasal tip. EFNB1 should be tested in TWIST1-negative clinically suspected Saethre-Chotzen syndrome to check for both Saethre-Chotzen syndrome and for CFNS [27,28].

Robinow-Sorauf syndrome also presents with a broad or duplicated hallux as well as mutations in the same TWIST gene. Therefore, Robinow-Sorauf is considered a milder form of Saethre-Chotzen syndrome [29-31].

Apert syndrome is a genetically inherited craniosynostosis syndrome characterized by various deformities in the skull, face and extremities [32]. Apert syndrome is autosomal dominant, due to p.Ser252Trp (c. $755 \mathrm{C}>\mathrm{G}$ ) in $71 \%$ of cases. The remaining minority of cases are due to p.Pro253Arg (c. $758 \mathrm{C}>\mathrm{G}$ ) missense mutation which transpires in the fibroblast growth factor receptor 2 (FGFR2) gene located on chromosome 10q26 [33]. Apert syndrome is rare with incidence of 1 in 65,000 to 200,000 births, affecting males and females equally [32,34]. There is predilection for incidence of this syndrome with increasing paternal age [35]. In addition to midface hypoplasia and symmetric syndactyly of the hands and feet, craniosynostosis is seen in all patients with Apert syndrome.

In comparison, the craniosynostosis seen in Apert syndrome is far more severe than that seen in Crouzon syndrome [36]. We can only theorize why extremities and cranial sutures are affected simultaneously in Apert syndrome, because there is not enough data to render this information complete in humans. Based on data on a murine model, the FGFR2 receptor lacks specificity and can bind other fibroblast growth factors, decreasing apoptosis of osteoblasts, resulting in syndactyly and craniosynostosis. However, despite this single murine model, the underlying mechanism remains unclear. What is clear however is that this process is linked to FGFR2 [37].

Intellectual development is greatly influenced by the timing of corrective antrostomy. Studies indicate about $50 \%$ of Apert syndrome cases report developmental delay [38]. The primary mode of treatment for craniosynostosis requires surgery to inhibit coronal suture closure and to preserve brain development.

It is valuable to consider the possible mechanisms by which fusion of the metopic sutures occurs to prevent future craniosynostosis formation during fetal development. This is a multi-faceted process with diverse etiologies and inciting factors. Surprisingly, a normal cephalic index (maximum cranium width/maximum cranial length) is observed in most cases of trigonocephaly; however, there is concurrent bitemporal shortening and biparietal broadening [39].

Complications of craniosynostosis include increased intracranial pressure and restriction in brain growth. The brain growth is hindered by the restriction of the cranial growth, and the brain is more likely to be compressed when many sutures are involved [40]. Vision, speech, and hearing impediments 


\section{Review Article}

are also common due to the compression of cranial nerves. It is indeterminate if much difference aside from psychomotor development and prevention of social isolation is made before and after surgical correction regarding the neurodevelopment in craniosynostosis patients. We theorize that de novo mutations, possible epigenetic changes due to environmental teratogens and increased parental age may lead to dysregulation of transcription factors involved in bone growth patterns and cranial suture fusion to cause what may be a spectrum of syndromes involving craniosynostosis.

Children present with higher rates of cognitive, behavioral, speech, and neurodevelopmental issues. Development is not always affected; however, developmental and intellectual delays can occur in children with trigonocephaly or other suture synostosis. Affected patients have behavioral disturbance, speech and language delay, impaired cognition, and exhibit symptoms seen in the following psychiatric disorders: attention deficit hyperactivity disorder (ADHD), oppositional defiant disorder (ODD), autism spectrum disorder (ASD), and conduct disorder (CD). Many of these problems typically become evident at school age. Calcification of the sutures may be induced earlier than normal due to oxidative stress on osteoblasts resulting from environmental toxins or postnatal ventilation given after a premature birth [40-43].

The Bayley Scales of Infant Development performed a study in fifteen children less than a year old two months before and one year after surgery [44]. These children had single-suture nonsyndromic craniosynostosis, and the mean score for mental development index were equivalent before and after surgery. In contrast, there was advancement in psychomotor development before and after surgical correction [44]. Surgical correction prevents intracranial hypertension and improves the child's self-esteem, as a craniofacial deformity can lead to social isolation. Nine to twelve months is the recommended age for surgical intervention, but patients should be evaluated as soon as they can, if possible, within the first weeks of life [44].

A trained social worker will inquire if the school is appropriate for the child's needs when the child is of school-going age. It is paramount to regularly screen for PTSD in children with craniosynostosis. Being away from home, separation from parents, being in pain, and being given intravenous therapy by people they may not be comfortable with yet are all risk factors for PTSD [45]. One out every ten children and parents develop PTSD after the child is admitted to the intensive care unit according to a study performed by Bronner [45]. If PTSD is suspected, the child must be referred to a psychologist. Adolescents who need surgical intervention need to be placed with a trained social worker. The craniofacial center that the family is working with needs to have an Early Intervention Program/peer supervision group in place to increase strength and support. Good communication between the craniofacial team and the Child Health Clinic, general practitioner, and pediatrician should be established. Pedagogical care is encouraged.

\section{CONCLUSION}

Summatively, there are several genetic syndromes that involve craniosynostosis and present with hydrocephalus or with learning and speech difficulties. A set of key genes and proteins have been identified to be involved through rpsblast. These syndromes have unique cranial features as well as psychiatric associations with ADHD, ODD, ASD and CD. There may be a spectrum of these syndromes rather than a set of distinct types of syndromes.

Looking forward, further work is necessary to address how transcription factor dysregulation leads to the accompanying classical anatomic features seen in the syndromes of craniosynostosis. Clinical refinement in the criteria of the syndromes involving craniosynostosis would appropriately allow for identification of patient severity and associated behavioral and psychiatric characteristics to better plan for future intervention. Earlier identification may lead to more appropriate inclusion of anticipatory surgical planning and educational interventions, prior to the development of behavioral, speech and psychiatric conditions, to improve the quality of life of these patients.

\section{REFERENCES}

1. NCBI Genbank: fibroblast growth factor receptor 2 (bacteriaexpressed kinase, keratinocyte growth factor receptor, craniofacial dysostosis 1, Crouzon syndrome, Pfeiffer syndrome, JacksonWeiss syndrome), isoform CRA_e [Homo sapiens] GenBank: EAW49356.

2. Venter JC. Adams MD, Myers EW, Li PW, Mural RJ, et al. (2001) The Sequence of the Human Genome. Science 291: 1304-1351.

3. Armand T, Schaefer E, Rocco FD, Edery P, Collet C, et al. (2019) Genetic bases of craniosynostosis: An update. Neurochirurgie 65: 196-201.

4. Perrine SMT, Wu M, Stephens NB, Kriti D, Bakel H, et al. (2019) Mandibular dysmorphology due to abnormal embryonic osteogenesis in FGFR2-related craniosynostosis mice. Dis Model Mech 12: dmm038513.

5. Timberlake AT, Jin SC, Nelson-Williams C, Wu R, Charuta GF, et al. (2019) Mutations in TFAP2B and previously unimplicated genes of the BMP, Wnt, and Hedgehog pathways in syndromic craniosynostosis. Proc Natl Acad Sci U S A 116: 15116-15121. 
6. Shimbo H, Oyoshi T, Kurosawa K (2018) Contiguous gene deletion neighboring TWIST1 identified in a patient with SaethreChotzen syndrome associated with neurodevelopmental delay: Possible contribution of HDAC9. Congenit Anom (Kyoto) 58: 33-35.

7. Sawh-Martinez R, Steinbacher DM (2019) Syndromic Craniosynostosis. Clin Plast Surg 46: 141-155.

8. Iida C, Sakamoto Y, Miwa T, Yoshida K, Kishi K (2019) Posterior Distraction First or Fronto-Orbital Advancement First for Severe Syndromic Craniosynostosis. J Craniofac Surg 30: 47-49.

9. Bessenyei B, Nagy A, Szakszon K, Mokanszki A, Balogh E, et al. (2015) Clinical and genetic characteristics of craniosynostosis in Hungary. Am J Med Genet A 167A: 2985-2991.

10. Harada A, Miyashita S, Nagai R, Makino S, Murotsuki J (2019) Prenatal sonographic findings and prognosis of craniosynostosis diagnosed during the fetal and neonatal periods. Congenit Anom (Kyoto) 59: 132-141.

11. Trench L, Bracken S, Mclnerney N, Caird J, Murray DJ (2019) OC7 Nonsyndromic craniosynostosis: referral patterns for metopic, coronal and lambdoid craniosynostosis in Ireland; 2009-2018, a 10-year review. Archives of Disease in Childhood 104: A3-A3.

12. Pournima G, Monica Y, Meghna S (2011) Crouzon syndrome: A case report. European J Dent Med 10: 1-5.

13. Meyers GA, Orlow SJ, Munro IR, Przylepa KA, Jabs EW (1995) Fibroblast growth factor receptor 3 (FGFR3) transmembrane mutation in Crouzon syndrome with acanthosis nigricans. Nat Genet 11: 462-464.

14. Di Rocco F, Biosse Duplan M, Heuzé Y, Kaci N, Komla-Ebri D, et al. (2014) FGFR3 mutation causes abnormal membranous ossification in achondroplasia. Hum Mol Genet 23: 2914-2925.

15. Snyder-Warwick AK, Perlyn CA, Pan J, Yu K, Zhang L, et al. (2010) Analysis of a gain-of-function FGFR2 Crouzon mutation provides evidence of loss of function activity in the etiology of cleft palate. Proc Natl Acad Sci USA 107: 2515-2520.

16. Ke R, Yang X, Tinayi C, Ge M, Lei J, et al. (2015) The C342R mutation in FGFR2 causes Crouzon syndrome with elbow deformity. J Craniofac Surg 26: 584-586.

17. Pal US, Gupta C, Chellappa AA (2012) Crouzon syndrome with primary optic nerve atrophy and normal brain functions: A case report. J Oral Biol Craniofac Res 2: 116-118.

18. Mathijssen IM (2015) Guideline for Care of Patients with the Diagnoses of Craniosynostosis: Working Group on Craniosynostosis. J Craniofac Surg 26: 1735-1807.

19. Rannan-Eliya SV, Taylor IB, De Heer IM, Van Den Ouweland AM, et al. (2004) Paternal origin of FGFR3 mutations in Muenketype craniosynostosis. Hum Genet 115: 200-207.

20. Xiaona Lu, Forte AJ, Steinbacher DM, Alperovich M, Alonso N, et al. (2020) Cranial Fossa Development in Differing Subtypes of Crouzon Syndrome. Journal of Craniofacial Surgery 31: 673-677.

21. Conrady CD, Patel BC (2019) Crouzon Syndrome. In: StatPearls [Internet]. Treasure Island (FL): StatPearls Publishing.

22. Sawh-Martinez R, Steinbacher DM (2019) Syndromic Craniosynostosis. Clin Plast Surg 46: 141-155.
23. Seki E, Enomoto K, Tanoue K, Tanaka M, Kurosawa K, et al. (2019) Tracheal cartilaginous sleeve in patients with BeareStevenson syndrome. Congenit Anom (Kyoto).

24. Lebland S, David D, Colley S, Buckley M, Roscioli T, et al. (2018) Atypical Skin Manifestations in FGFR2-Related Craniosynostosis Syndromes Broaden the Phenotypic Spectrum. Mol Syndromol 9: 149-153.

25. Sewda A, White SR, Erazo M, Hao K, Garcia-Fructuso G, et al. (2019) Nonsyndromic craniosynostosis: Novel coding variants. Pediatr Res 85: 463-468.

26. Kent WJ, Sugnet CW, Furey TS, Roskin KM, Pringle TH, et al. (2002) The human genome browser at UCSC. Genome Re 12: 996-1006.

27. Lee E, Le T, Zhu Y, Elakis G, Turner A, et al. (2017) A craniosynostosis massively parallel sequencing panel study in 309 Australian and New Zealand patients: Findings and recommendations. Genet Med 20: 1061-1068.

28. Howaldt A, Nampoothri S, Yesodharan D, Udayakumaran S, Subhash P, et al. (2019) Four novel mutations in EFNB1 in Indian patients with craniofrontonasal syndrome. J Hum Genet 64: 867-873.

29. Chun K, Teebi AS, Jung JH, Kennedy S, Laframboise R, et al. (2002) Genetic analysis of patients with the Saethre-Chotzen phenotype. Am J Med Genet 110: 136-143.

30. Czerwinski M, Kolar JC, Fearon JA (2011) Complex craniosynostosis. Plast Reconstr Surg 128: 955-961.

31. Kress W, Schropp C, Lieb G, Peterson B, Busse-Ratzka M, et al. (2006) Saethre-Chotzen syndrome caused by TWIST 1 gene mutations: Functional differentiation from Muenke coronal synostosis syndrome. Eur J Hum Genet 14: 39-48.

32. Kutkowska-Kaźmierczak A, Gos M, Obersztyn E (2018) Craniosynostosis as a clinical and diagnostic problem: Molecular pathology and genetic counseling. J Appl Genet 59: 133-147.

33. Işık E, Atik T, Onay H, Ozknay F (2017) Two patients with Apert syndrome with different mutations: the importance of early diagnosis. Turk pediatri arsivi 52: 231-235.

34. Cohen MM, Kreiborg S, Lammer EJ, Cordero JF, Mastroiacovo P, et al. (1992) Birth prevalence study of the Apert syndrome. Am J Med Genet 42: 655-659.

35. Goriely A, Mcvean GAT, Rojmyr M, Ingemarsson B, Wikie AOM, et al. (2003) Evidence for selective advantage of pathogenic FGFR2 mutations in the male germ line. Science 301: 643-646.

36. Conrady CD, Patel BC, Sharma S (2020) Apert Syndrome. In: StatPearls [Internet]. Treasure Island (FL): StatPearls Publishing.

37. Hajihosseini MK, Duarte R, Pegrum J, Donjacour A, Lana-Elola E, et al. (2009) Evidence that Fgf10 contributes to the skeletal and visceral defects of an Apert syndrome mouse model. Dev Dyn 238: 376-385.

38. Renier D, Arnaud E, Cinalli G, Sebag G, Zerah M, et al. (1996) Prognosis for mental function in Apert's syndrome. J Neurosurg 85: 66-72.

39. Neurological consequences of Prenatal, Perinatal and Early Postnatal Interference with Brain Development.

40. https://files.eric.ed.gov/fulltext/ED368492.pdf 


\section{Review Article}

41. Mendonca DA, White N, West E, Dover S, Solanki G, et al. (2009) Is there a relationship between the severity of metopic synostosis and speech and language impairments? J Craniofacial Surgery 20: 85-89.

42. Kilcoyne S, Luscombe C, Scully P, Jayamohan J, Magdum S, et al. (2019) Language Development, Hearing Loss, and Intracranial Hypertension in Children with TWIST1-Confirmed SaethreChotzen Syndrome. J Craniofac Surg 30: 1506-1511.
43. Paul T, Makkar SS, Mohan S, Mohan P (2020) Metopic Craniosynostosis and Hydrocephalus in a Premature Opioid Dependent Baby. Ann Child Neurol 28: 167-168.

44. Overview of craniosynostosis.

45. Mathijssen I (2015) Guideline for Care of Patients with the Diagnoses of Craniosynostosis: Working Group on Craniosynostosis. J Craniofac Surg 26: 1735-1807.

Citation: Singh-Makkar S, Grewal TK, Kaur J, Sharma A, Paul T, et al. (2021) The Genetics and Learning Disorders of the Syndromes Involving Craniosynostosis. Appl Cell Biol, 9(2), 2021 [49-54] 